\title{
Vladimir Solovjovs mystisches Erlebnis
}

\author{
Von MARIA WIDNÄS
}

Vladimir Solovjov gehört immer noch zu den rätselhaftesten Erscheinungen des geistigen Lebens Russlands. Der Grund dafür ist vor allem, dass die idealistische Philosophie, welche er in den 7oer Jahren des vorigen Jahrhunderts als Gegensatz zu dem damals herrschenden Rationalismus schuf, ganz auf seinem mystischen Erlebnis begründet ist. Erst aus einem Studium der eigenartigen seelischen Struktur Solovjovs und seiner mystischen Vision der Weisheit Gottes, der Sophia, ergibt sich der Schlüssel zu seinem Schaffen als Philosoph und als Dichter.

Vladimir Solovjov tritt uns auf einem vom Künstler Jarošenko gemalten Jugendporträt ${ }^{1}$ als ein ausgesprochen ukrainischer Typus entgegen. Er glich sehr seiner Mutter, die den ukrainischen Philosophen Skovoroda unter ihre Ahnen zählte. Sein Gesicht ist schmal und lang, mit zurückfliehendem Kinn und Stirn. Er hat grosse, traumhaft verschleierte, dunkelgraue Augen. Der in die Ferne gerichtete, schwärmerische Blick ist von den Zeitgenossen als der Blick eines Propheten bezeichnet worden. Nach der Bestimmung Kretschmers vertritt er wohl den sog. schizothymen Gesichtstypus². Typisch ist denn auch, dass Solovjov schon mit 47 Jahren an allgemeiner Erschöpfung des Organismus ,als Greis “' ${ }^{\prime 3}$ gestorben ist. Solovjov kann nach Kretschmer als ,,ein pathetischer Idealistentypus“ bezeichnet werden ,der ein Vermögen zum tragischen Erlebnis hat", ein Typus der, um mit Kretschmer zu reden oft ,,an Hyperästhesie mit eingeschränktem Gefühlskreis und daraus entspringend autistischer Unfähigkeit zur objektiven Registrierung der Wirklichkeit" leidet und ,eine entschiedene Hinwendung zum Unwirklichen, zum Ideal, zur Abstraktion, zum Schönen und Gedankenvollen, zum Aufbau einer abgeschlossenen, zarten Innenwelt“ und hieraus erwachsener „elek-

\footnotetext{
${ }^{1}$ Vladimir Solov'ev, Stichotvorenija, 6. Aufl., Moskva I9I 5, S. 2.

${ }^{2}$ Ernst Kretschmer, Körperbau und Charakter, 2 r/22 Aufl, Heidelberg 1955, S. 209.

${ }^{3}$ Ekaterina El' cova, „,Sny nezdešnie“, Sovremennyja Zapiski 28, 1926, S. 272.
} 
tiver schwärmerischer Zuneigung zu einzelnen Personen " besitzt ${ }^{1}$. Die Charakteristik Kretschmers scheint nicht nur gut auf Vladimir Solovjov zu passen, sondern auch auf alle seine Geschwister. Denn das Verhältnis der Solovjovs zum Leben und zu einander hatte etwas ausgesprochen Überschwengliches und Überspanntes, berichtet die Freundin der jüngsten Schwester Vladimirs, E. El'cova, in ihren Erinnerungen "Sny nezdešnie“ (Jenseitsträume). ,Alle Solovjovs“, sagt sie, ,,waren nervös und diese Nervosität kam oft zum lärmenden Ausdruck. Alle Solovjovs neigten zur Schwermut“"2. Mit grösster Begeisterung fürs Leben wechselten Lahmheit, Langeweile und Verzweiflung ab3. Die scheinbare Oberflächlichkeit ihres Lebens und Treibens hatte sicher ihren Grund in einer (nach Kretschmer) ,,unlogischen Weise die Einheitlichkeit des Seelenlebens zu suchen "4. Die persönliche, ererbte Anlage und die ganze Umgebung prädestinierten Vladimir Solovjov zum Mystiker. Und dazu kam auch noch Vladimirs Fähigkeit zum ,eidetischen Erlebnis" ${ }^{\prime}$, welche den übrigen Geschwistern gefehlt haben soll. Ein Insichversinken befiel Vladimir Solojov oft schon als Kind und auch später als Erwachsener mitten im gesellschaftlichen Gespräch. Er war der Meditation und vielleicht auch der inneren Nachbildung der Vorstellungen nicht zu entreissen, wenn diese Nachdenklichkeit ihn befiel. Die erste nachbildende, eidetische Vision hat er seiner eigenen Angabe nach als neunjähriger Knabe in der Kirche am Himmelsfahrtstag gehabt, und ihr ist er sein Leben lang treu geblieben. Viel später, nach $3^{6}$ Jahren, beschreibt er diese Vision halb scherzhaft im Gedichte „Drei Begegnungen“ mit folgenden Worten:

Zum ersten Mal - ich glaub' es sind vergangen

Seitdem wohl volle sechsunddreissig Jahr -

Dass meine Kinderseele jäh umfangen

Von Liebesgram und wirren Träumen war.

Neun Jahr war ich, und sie zählt auch neun Jahre.

,,Es war ein Maientag in Moskau“, sagte Phöt.

Da hab ich mich erklärt ... sie schwieg ... o Gott,

Gewiss ein andrer schon ... Wenn ich erfahre ...

1 Kretschmer, S. 209.

${ }^{2} \mathrm{El}^{\prime}$ cova, S. 240.

${ }^{3}$ Ibid., S. 237.

${ }^{4}$ Kretschmer, S. 209 u. ff.

5 E. R. Jaensch, Die Eidetik, Leipzig I927, S. 33-34, 67. 
Duell, Duell' ... Ein Gottesdienst am Morgen

Von Himmelfahrt ... Mein Herz mit Liebe rang ...

Da tönts: ,,O lasset dieses Lebens Sorgen“ ...

Lang dehnte sich und zitternd starb der Klang.

Es steht der Altar offen, doch verschwunden

Sind Priester und das Volk mit einem Mal,

Die Liebesqual ist völlig überwunden,

Azur in mir, ringsum und überall.

Durchleuchtet von den Strahlen, goldnen, blauen,

Standst Du vor mir mit hellem Angesicht,

Du hältst die Blume aus den Himmelsauen,

Und winktest lächelnd mir und schwandst in Licht.

Es losch in mir des Kindes Liebesflamme,

Die Seele war für dieses Leben blind,

Doch traurig klagte meine deutsche Amme:

„Wolodenka, sie ist ein dummes Kind."1

Der Neunjährige vermag sich über die unverstandene Kinderliebe mit der Vision einer schönen, ihm wohlgesinnten himmlischen Freundin zu trösten und vergisst darüber den unschönen, grauen Alltag, vergisst die Bosheit der Menschen.

Obschon die erste Vision von Solovjov erst im Mannesalter und in einem selbstironischen Gedicht niedergeschrieben worden ist, können wir sie für ein Zeugnis der ausgesprochen eidetischen Veranlagung Vladimir Solovjovs halten. Von der Fähigheit, sein Ideal leiblich vor sich zu sehen, stammt seine Wirklichkeitsfremdheit. Man kann sich denken, dass die stark beleuchtete Kirche mit ihren Ikonen, die in Gold und Blau schimmerten, ganz natürlich die goldumstrahlte Vision hervorrief. Aus dem Inneren heraus projiziert stand aber die wunderschöne Frau vor dem Jungen und winkte ihm freundlich zu. Die Blume in der Hand kann eine spätere Zugabe sein. Der strahlende Blick der blauen Augen ist aber ein ständiges Attribut der Erscheinung, das Solovjov auch später ganz der grauen Wirklichkeit entrückt und ihn zum ,Dummen Ding“ macht.

Der erste Eindruck der lichten Gestalt verblasste ganz und wurde vergessen als Solovjov in die Schule und später in die Universität eintrat. Dies war die Zeit der Alleinherrschaft des Materialismus im Russland der 6oer Jahre. Die Materie allein war der Abgott dieser Zeit. Aus ihr sei alles entstanden.

${ }^{1}$ Wladimir Solowjew, Gedichte von Wladimir Solowjew. Ins Deutsche übertragen von Dr. Kobilinski-Ellis und Richard Knies, Wiesbaden I925, S. 42-43. 
" Zu ihr gehe alles zurück. Zunächst hatte diese neue Lehre auf die Gemüter befremdend gewirkt. Vladimir Korolenko erzählt in seinen Erinnerungen „Istorija moego Sovremennika“"(Die Geschichte meines Zeitgenossen) wie schauerlich es einem vorkam, als alles vom Materialismus entblösst wurde. „Es war mir, als ob kalte Schneeflocken mir plötzlich auf den nackten Körper niederfielen"1, sagt Korolenko. Man gewöhnte sich aber an den nackten Materialismus und wurde sogar begeistert dafür, alles Geheime und Traditionelle zu entschleiern, wie es z. B.Černyševskij und Dmitrij Pisarev² öffentlich taten, bis, endlich, in den 7oer Jahren die russischen Jünglinge gewahr wurden, dass durch Materialismus und Verneinung aller Tradition nicht so viel gewonnen war, und der Abgott Materie gestürzt wurde. Auch Vladimir Solovjov war begeisterter Materialist in seinen Gymnasialjahren, als er zusammen mit seinem Freund Lopatin die Kreuze auf dem Friedhof umwarf und mit Füssen trat ${ }^{3}$. Noch war er Materialist, als er sich ,wie alle tüchtigen Menschen" ${ }^{4}$ in die Naturwissenschaftliche Fakultät an der Moskauer Universität einschreiben liess. Dann aber, nach vier ziemlich erfolglosen Jahren, kam auch für ihn die grosse Enttäuschung an dem Materialismus. Solovjov hatte, wie alle übrigen, einen Gott in der Materie gesehen. Es erwies sich aber, dass die Materie nicht einmal etwas Einheitliches bot, sondern nur ein Chaos von Atomen. Kein Zusammenhang liess sich mit Hilfe der Naturwissenschaften im Weltall entdecken. So verliess denn Solovjov die Naturwissenschaftliche Fakultät und ging zu den Humaniora über"4.

Der Übergang muss ein schmerzlicher gewesen sein. Solovjov spricht in seinem Brief an die Kusine von ,,der Zeit der Verzweiflung““5. Er suchte nach

1 Vladimir Korolenko, Istorija moego sovremennika, Sanktpeterburg i 909 , S. 430.

2 A. Černyševskij, ,Antropologičeskij princip v istorii“", Polnoe sobranie sočinenij, tom 8, Sankt Peterburg I906; Dm. Pisarev, Sočinenija, tom I-6, Sankt Peterburg I 894 .

${ }^{3}$ S. M. Lukjanov, O Vl. S. Solov'eve v ego molodye gody, Kniga 3: Vypusk I, Petrograd I 921, S. 83.

${ }^{4}$ S. M. Lukjanov, ,,O V1. S. Solov' eve v ego molodye gody“" Z̆urnal Ministerstva Narodnago Prosveščenija 1915:7, S. 33-34; Pis'ma Vladimira Sergeeviča Solov'eva, tom 3. Pod red. E. L. Radlova, S.-Peterburg I9 I , S. 64. Hierbei zitiert Solov'ev in russischer Übersetzung die Worte Fausts ,Geheimnisvoll am lichten Tag, lässt sich Natur des Schleiers nicht berauben, und was sie deinem Geist nicht offenbaren mag, das zwingst du ihr nicht ab mit Hebeln und mit Schrauben" Faust I. Nacht. Faust.

${ }^{5}$ L. Lopatin, ,,V1. Solov'ev“, Voprosy filosofii i psichologii, 24, Kniga I 19, S. 356359; Vladimir Solov' ev, Pis' ma, tom 3, S. 75. 
dem Sinn des Lebens als er sich von der empirischen Welt abwandte und suchte leidenschaftlich. Zunächst wandte er sich Leibnitz und dessen Monadenlehre zu. Dann fing er an, mit Schopenhauer wie mancher andere an der Wirklichkeit des Daseins zu zweifeln und verfiel dem Pessimismus dieses in den 7 oer Jahren in Russland schon viel gelesenen Philosophen. Unter der Leitung seines Universitätslehrers, des Professors der Philosophie Pamphil Danilovič Jurkevič ${ }^{1}$, der ein ausgesprochener Verfechter der Ideenlehre Platos war, ging Solovjov bald zum Platonismus über. $\mathrm{Zu}$ dieser Zeit, als er seine Studien an der Universität beendete, schrieb Solovjov eines seiner ersten Gedichte, welches das von Plato entworfene Bild der in einer dunklen Grotte gefesselten Menschen widerspiegelt:

$V$ sne zemnon my teni, teni

Žizn' igra tenej

Rjad dalekich otraženij

Večno svetlych dnej
Im Lebenstraum wir sind nur Schatten, Das Leben Schattenspiel,

Bloss ein Spiegelbild von fernen

Ewig, hellen Tagen

Von Plato, der bei dem Spiegelbild des Spiegelbilds der Idee verharrt, weicht aber Solovjov schon jetzt ab. Denn Solovjov glaubt fest daran, dass der Sonnenaufgang für die Menschheit schon naht. Je finstrer die Nacht, desto unklarer die Umrisse der falsch geordneten Wirklichkeit. Bald wird dieser Wahn, von dem wir gefesselt sind, verschwinden, sich auflösen, und ein neuer Tag, eine helle, harmonische Wirklichkeit wird aus der Finsternis emporleuchten. „Traure nicht", sagt Solovjov zum Schluss, „bald naht der ewige, helle Tag'"2.

$\mathrm{Zu}$ dieser Zeit glaubte Solovjov ganz fest daran, dass, wenn nur alles Bestehende zerstört werde, die Wahrheit der Welt ihre eigenen Gesetze auferlegen würde, welche mit der vernüftigen Idee des Daseins übereinstimmen und jeden glücklich machen würden. Er nennt sich selbst einen Anarchisten in einem fingierten Gespräch mit seiner Kusine und mit einem jungen Mediziner. Alles muss sobald wie möglich zerstört werden, damit alles wieder gut werdes. Deshalb konnte Solovjov zu dieser Zeit hell auflachen, wenn er erfuhr, dass es den Leuten in dieser materiellen Welt schlecht

\footnotetext{
1 Pamfil Danilovič Jurkevič, Professor der Philosophie an der Moskauer Universität seit I86I, polemisierte im Jahre I 862 mit Černyševskij gegen die Behauptung, dass das Organische aus dem Unorganischen entstehe.

${ }^{2}$ S. M. Lukjanov, ,O Vl. S. Solov'eve“, S. 6r.

${ }^{3}$ Vl. Solov'ev, ,Na zare tumannoj junosti“, Solov'ev, Pis'ma 3, S. 294. 
ging. ,,Je schlechter, desto besser"1, war sein Lieblingsausdruck zu dieser Zeit. Deshalb lacht er manchmal über sich selbst, wenn er einsieht, dass er den gewöhnlichen Alltagsmenschen mit seinen Ideen von einer neuen, ideellen Weltordnung, als ein verrückter Fabulierer vorkommen muss, wovon u. a. die von ihm über seine eigene Bestrebungen verfasste Satire „Die weisse Lilie“ zeugt².

Der ganz bestimmte Glaube an einen neuen Schöpfungstag war eine Gewissheit für Solovjov schon, als er in den 7oer Jahren seine „Krisis der westlichen Philosophie" vollendet hatte. Er fertigte die westliche Philosophie $\mathrm{ab}$ als ein Suchen nach rationeller Erklärung des Weltalls, das verfehlt war. Selbst wollte er eine Einheitsphilosophie begründen, die konkreter als diejenige eines Schelling oder eines Ed. v. Hartmann sein sollte, indem sie Materie und Geist in einer neuen Weise vereinigen sollte. Ungeschmälert sollten beide, Geist und Materie, sich verkörpern, so wie er es selbst geschaut hatte. Diese Idee der neuen Einheit hatte Solovjov in seiner Diplomarbeit (,Der mythologische Prozess im Altheidentum“3) über die Entwicklung des religiösen Bewusstseins der Völker vom Monotheismus, über Polytheismus zum einzigen Gott schon entworfen. Er suchte aber jetzt nach uralten Lehren, die schon dasselbe Reich der Alleinheit vor Augen gehabt hatten. Um vor allem die Gnosis zu studieren, begab er sich nach London. Im British Museum war er von der einzigen Idee dieser Alleinheit besessen. Dies kommt zum Ausdruck in der Schilderung der zweiten Begegnung mit der lichten, hehren Frau seiner Träume, die er als Knabe gesehen hatte, die sein Lesen überwacht und dafür sorgt, dass ihn nichts veranlässt, sich von ihr abzuwenden:

„Bin ich allein im Lesesaal gewesen, Ich träumte, Gott sei Zeuge, nur von Ihr, Was alles über Sie ich sollte lesen, Von selbst geheime Kräfte wählten's mir.

Wenn aber nicht mit allerreinstem Herzen

Ein Buch ich ausgesucht im Lesesaal, Die seltsamsten Geschichten, böse Scherze Zurück nach Haus mich trieben jedesmal."

\footnotetext{
1 S. M. Lukjanov, $O V l$. S. Solov'eve, t. 3, S. 129.

${ }^{2}$ Vladimir Solov'ev, ,Belaja lilija“, Pis'ma 3, S. 299-335.

${ }^{3}$ Vladimir Solov'ev, ,,Mifologičeskij process $\mathrm{v}$ drevnem jazyčestve“ Pravoslavnoe Obozrenie, I873:1 I, S. 635-665.
} 
Da erscheint ihm das Antlitz der hehren Frau:

, Und einmal endlich (als schon fühlbar waren

Die Herbstesschatten) sagte ich zu Ihr:

Du bist ja hier, warum seit Kindheitsjahren

Du Blume Gottes, bliebst unsichtbar mir?"

Kaum dass ich dieses Wort auch erst nur dachte,

Da überstrahlte alles blaues Licht,

Und wieder stand Sie vor mir, Glanz entfachte

Ihr Antlitz nur, ihr helles Angesicht."

Die Vision befiehlt Solovjov: ,Auf nach Ägypten!“ Ganz unerwartet für die ganze Familie, die er in Moskau zurückgelassen hatte, bricht Solovjov auf und begibt sich nach Kairo. Für ihn selbst war die Abreise vielleicht doch nicht unerwartet. Er hatte über Gnosis das gelesen, was das British Museum zu bieten hatte. Er hatte wahrscheinlich auch einen Hinweis auf Ägypten gefunden, vielleicht auf die Wüste und das sog. ,,Taborlicht", das Gnostiker und Heilige Väter gesehen hatten². Es war klar, dass er jetzt nach Ägypten musste, um an die Quelle der gnostischen Lehre zu kommen. Dies kommt nun anschaulich zum Ausdruck in der zweiten Vision, deren Echtheit für Solovjov wir übrigens nicht zu bezweifeln brauchen.

In Kairo, im Hotell Abbat angekommen, begibt sich Solovjov eines Abends hinaus in die Wüste, um die Lichterscheinung selbst zu erfahren. Das Unternehmen ist sehr gewagt, denn in der Wüste wimmelt es von Arabern. Diese umringen auch bald den Philosophen und binden ihn an Händen und Füssen, lassen ihn aber bald wieder los und verschwinden. Solovjov glaubt, er mag den Arabern in seinem Zylinderhut vielleicht als ein Teufel erschienen sein. Dies überstand er und war nun ganz allein in der dunklen Nacht in der Wüste. Es wurde kalt. Ein Schakal heulte in der Ferne. Da hörte er eine Stimme sanft zu ihm sagen: „Schlaf ein, mein armer Freund.“ Er schlief ein, und als er erwachte, war es kurz vor Sonnenaufgang. Da erschien ihm die ganze Herrlichkeit der Weltseele, der Königin des Alls:

,,Und wachte auf und sah - es atmen tief

Der Himmelskreis, die Erde, voll von Rosen.

Mit Augen voll Azur und Flammen überall

Im Himmelspurpur, wo dein Ruf noch wehte,

1 Wladimir Solowjow, Gedichte. Übertragen von Kobilinski-Ellis, S. 44; Solovjev soll im British Museum hauptsächlich die Kabbala gelesen haben, Lukjanov, O V1. S. Solov'eve, S. I43.

2 Siehe Edsman, oben, S. I2. 
Schautst Du mich an, als erste Morgenröte Des Schöpfungstags, als ewiges Weltenall.

Was war, was ist, was wird in Ewigkeiten,

Umschloss mein fester Blick in einem Kreis:

Ich sehe unten Meeres blaue Weiten,

Den fernen Wald und hoher Berge Eis.

Ich Alles schau, und Alles ist nur Eines,

Das Unermessne fand Dein hohes Mass ...

O Schönheitsbild, jungfräuliches und reines;

Vor mir, in mir nur Deinen Blick ich fass.

- - -

Ein Augenblick und sie verschwand, die Helle,

Der Sonnenball sich in die Höhe schwang,

Die Wüste schwieg, es betete die Seele,

Die ganze Welt war Ruf und Glockenklang ${ }^{1}$.

Das Gefühl des Humors verliess auch nach der Vision Solovjov nicht auf einen Augenblick. Er verstand, dass er den Gästen des Hotels Abbat in Kairo ganz toll erscheinen musste und beschrieb, wie ein alter General ihn dazu ermahnte, über sein Abenteuer zu schweigen, um nicht als Dummian zu gelten, denn „,soweit ist nicht gediehn der Menschen Klugheit, Schattierungen des Irrsinns zu verstehen“" Die Seele Solovjovs ist aber „umflossen vom blauen Dunst", denn er hat ,der Gottheit Angesicht" schauen dürfen. Deshalb sagt er am Schluss:

Den Wahn der Zeit so niederringend

Besiegte ich im Lebenslicht den Tod, Die Scheu im Liede fromm bezwingend, Verwob ich dich dem ew'gen Morgenrot ${ }^{3}$.

Solovjov hatte also, wie er meinte, das ,Angesicht der Gottheit" in der Wüste geschaut. Er identifiziert diese Gottheit erstens mit der „Weisheit Gottes“, die laut Salomo (Die Sprüche 9:r) ,sich ein Haus aufbaute, das mit sieben Säulen gestützt wurde," worüber auch Solovjov ein neues Gedicht verfasst. Zur gleichen Zeit oder gleich nachher, im Jahre 1876 , schreibt er auch ein förmliches Gebet an diese Gottheit nieder, die er „An-Soph, Jah, Soph-Jah" nennt, ein Gebet ,über die Offenbarung des Geheimnisses“. Es lautet wie folgt: ,Mit dem unaussprechbaren, furchtbaren und allmächtigen

1 Wladimir Solowjow, Gedichte. Kobilinski-Ellis, S. 48 .

2 (Wladimir Solovieff), Gedichte von Wladimir Solovieff, Übertragen von Marie Steiner, Dornach, Schweiz, 1949, S. 34.

3 Ibid., S. 35 . 
Namen beschwöre ich die Götter, die Dämonen, die Menschen und alles, was lebt. Schliesset zusammen die Strahlen eurer Kräfte, haltet ein die Quelle eures Willens und beteiliget euch an meinem Gebet. Auf dass wir die reine Taube Sions einfangen könnten, auf dass wir die kostbare Perle Ophirs bekämen, und damit Rosen und Lilien sich in der Wüste Sarons vereinigen möchten. - Allerheiligste Göttliche Sophia, Urbild der Schönheit und Süsse des über allen seienden Gottes, lichter Körper der Ewigkeit, Seele der Welten und einzige Königin aller Seelen, ich beschwöre dich aus unsagbarer Tiefe und durch die Gnade deines ersten Sohnes, des höchstgeliebten Jesus Christus: steige hinab in das Gefängnis der Seele, erfülle unsere Finsternis mit deinem Licht, zerschmelze die Fesseln unseres Geistes mit dem Feuer deiner Liebe, gib uns Licht und Willen, erscheine uns als sichtbar und wesentlich, verkörpere dich in uns und in der ganzen Welt, baue auf die verschwundene Fülle der Zeiten, damit die Tiefen bemessen würden und Gott Alles in Allem sei!" 1

Dieses Gebet zeigt sehr deutlich, dass Solovjov die Weisheit im Sinne der Gnostiker auffasst. In demselben Sinne verfasst er zur selben Zeit auch den „Gesang der Ophiten“"2, der die Riten dieser Sekte schildert, die ,weisse Lilie mit roten Rosen mischen" und die „Taube im Schoss des Uralten Drachens" erschauen. Diese Symbole „Lilien und Rosen“" und „Taube“ und „Drache“ (das Böse) kommen seitdem immer wieder in der Lyrik Solovjovs vor. Es ist das Bild der Himmelskönigin, das immer wiederkehrt und nach Kairo in dem Gedicht über die Königin, (die Weisheit Gottes, wie in den Sprüchen Salomos geschildert) sich ein Schloss, das auf sieben Säulen ruht, erbaut hat. ,Die Gebieterin mein thront im herrlichsten Schrein - sieben goldene Säulen sind sein" ${ }^{\prime 3}$, schreibt Solovjov. Die Königin gibt sich aber nicht zufrieden mit der Fülle von Rosen und Lilien in ihrem Garten und den silbernen Quellen, die ihn durchströmen. Sie ist voll Kummer. Denn ihr Freund versagt im fernen Norden einsam im Kampf gegen die böse Macht der Finsternis. Sie eilt zu ihm, und mitten im finstren Winter fühlt er das Wehen des Frühlings und sie flüstert $\mathrm{ihm}$ Worte des Trostes zu․ Während

\footnotetext{
1 Vladimir Solov'ev, Stichotvorenija, S. 294-295.

${ }^{2}$ Ibid., S. 65-66.

3 Ibid., S. 63.

${ }^{4}$ Ibid., S. 79 .
} 
seines ganzen Lebens empfindet Solovjov zu gewissen Zeiten die Anwesenheit der „Ewigen Freundin“, der Sophia. Diese Offenbarungen, die mit der dritten Begegnung in Kairo anfangen, sind in mehreren Gedichten Solovjovs zum Ausdruck gekommen. Schon I 875 schreibt er in Kairo nieder:

„Ganz in hellblauem Lichte erschien mir heute Morgen die Königin mein — Süsse Schauer erfüllten das Herz mir — Und im Schimmer des nahenden Tages - Goss ihr Licht sich mir hell in die Seele. - Trüb und qualmend verglomm in der Ferne - Böse flammend das irdische Feuer" ${ }_{11}$. Die lichte Erscheinung offenbart sich dem Dichter inmitten der heissen Windwirbel in der Wüste als er sie schon beinahe vergessen hatte und trägt seinen Geist empor zum Himmel. Später empfindet er während des lichten Sommertages die Einheit von Erdenleben und Himmel (,O Erde, Herrscherin“)2. Als der Tag heiss und doch neblig ist, erwartet der Dichter die Offenbarung des Angesichts der „Ewigen Freundin“, das, ,wie die Sonne“ über allem Kummer aufstrahlen wird. Während Solovjovs Aufenthalt in Finnland Anfang der goer Jahre, erscheint ihm mitten im schneebedeckten Walde „,das blaue Auge“ der „Ewigen Freundin" im Nebel und blickt ihn an mit der Verheissung ,,das Unerwartete wird bald geschehen" ${ }^{\prime 3}$. Endlich beschwört er im Archipelag die Meerdämonen mit dem strahlenden Bild des Ewig-Weiblichen, der Aphrodite, die sie einstmals schon besiegt hat, welche die ,Jungfrau der Pforte des Lichts" ist4. Wo der Dichter auch sei, ,tags und nachts" fühlt er auf einmal, dass er nicht allein ist, dass ein strahlendes Augenpaar ihm , in die Seele schaut"; er empfindet wie der kalte Winter sich in Sommer verwandelt, wie alles Licht und Wasser wird, wo sich der unvergessliche Blick spiegelt, der die Zeitlosigkeit bedeutet, in der alle Lebenstage zusammenfliessen"5. Das mystische Erlebnis ist zum Glaubensbekenntnis Solovjovs geworden. Es ist für ihn stets gegenwärtig, und er fühlt sich verpflichtet, ihm treu zu bleiben und es der Welt zu verkündigen. Dies tut er vor allem in dem neuen System, das er aufbaut, als er „Die Prinzipien des integralen Wissens" nach seiner Rückkehr aus Kairo niederschreibt.

\footnotetext{
1 Gedichte von Wladimir Solovieff ... Marie Steiner. S. I5.

2 Vladimir Solov'ev, Stichotvorenija, S. 89.

3 Ibid., S. I 50.

4 Ibid., S. I 8 I-I 85 .

${ }^{5}$ Ibid., S. 203.
} 
„Die Philosophie“ sagt Solovjov"1, „ist vor allem die Antwort auf die Frage nach dem Ziel des Lebens, des Seins“. Die Menschheit ist nach ihm ein lebendiger Organismus und besitzt potentiell die Möglichkeit, verschiedene Seiten des Seins zu entwickeln. Sie entwickelt sich und wächst übrigens ganz wie die Pflanze sich allmählich aus dem Keim entwickelt. Am Anfang sind nur die potentiell vorhandenen Möglichkeiten der Entwicklung da, als die Pflanzen noch kein Bewusstsein von sich selber haben. Sobald sie sich zu verschiedenen Elementen entfalten, behaupten sie sich als unabhängige Wesen gegenüber dem Undifferenzierten, Primären, aus welchem sie stammen. Bei der dritten Stufe der Entwicklung angelangt, empfinden sie aber bewusst ihre Zugehörigkeit zum Urquell, zum Ganzen. In verschiedenen Sphären, von denen Solovjov drei unterscheidet, manifestieren sich die drei Grundkräfte des Menschen: der Wille, das Gefühl und das Wissen. Unter diesen drei hebt Solovjov vor allem das Gefühl hervor, welches sich im Bereich der Mystik betätigt und grundlegend für jedes Schaffen ist. Es mag, gibt er zu, wunderlich erscheinen, dass der Mystik eine so bedeutende Rolle zugewiesen wird. Die Bedeutung der Mystik ist aber unstreitig, wie auch diejenige des Gefühls, auf dem sie fusst, so gross, weil das Gefühlserlebnis, das mystische Erlebnis also, nach Solovjov (im Gegensatz zu Wille und Wissen) der einzige Bereich ist, wo der Mensch in Berührung mit der Fülle des Seins treten kann, welche in einer transzendenten Welt für sich selbst existiert (und immer existiert hat), welche Ursprung alles Beseelten und alles Schaffens (vor allem des künstlerischen Schaffens) ist. Denn Schaffen springt aus dem Quell des Gefühls, nicht aus dem des Denkens oder des Willens hervor und hat zum Ausdruck die Phantasie (nicht den Verstand oder die praktische Aktivität). Schaffen und mystisches Gefühlserlebnis sind übrigens beide von einer Extase hervorgerufen.

Es ergibt sich für das holistische Weltall folgendes Schema, das in den „Prinzipien des integralen Wissens" von Solovjov vorgeführt wird":

1 Vladimir S. Solov' ev, ,,Filosofskija načala cel'nago znanija“, Vladimir Sergeevič Solov'ev, Sobranie sočinenij pod red. i s primečanijami S. M. Solov'eva i E. L. Radlova, 2-e izd., tom I, S.-Peterburg I9II, S. 250 u. ff.

2 Ibid., S. 264. 
Sphäre des Schaffens

Subj. Ausgangspunkt

Gefühl

Obj. Prinzip

Schönheit
Sphäre des Wissens

Subj. Ausgangspunkt

Gedanke

Obj. Prinzip

Wahrheit
Sphäre der praktischen Aktivität

Subj. Ausgangspunkt

Wille

Obj. Prinzip

Das Gute

Die Verwirklichung dieser Prinzipien geschieht auf den verschiedenen Gebieten auf drei verschiedenen Stufen, die sich aber gegenseitig berühren:

\section{Sphäre des Schaffens}

I. Stufe (absolute) Mystik

2. (formale) Kunst

3. (materielle) Technisches Können und Kunst

Bereich des Wissens

I. Stufe: Theologie

2. Abstr. Philosophie

3. Positive Wissenschaft

\section{Bereich des Heils}

I. Stufe: Geistige Gemeinschaft. Kirche

2. Politische Gemeinschaft (Staat)

3. Wirtschaftliche Gemeinschaft (Landstaat)

Keines der Elemente der drei Stufen kann sich gegenüber den beiden anderen derselben Stufe als wichtiger oder weniger wichtig behaupten. Dagegen empfindet man die Zusammengehörigkeit und Unterordnung der drei Stufen untereinander im Bereich der Schönheit, der Wahrheit oder des Guten. Die drei Stufen gehören unbedingt zusammen und ergänzen einander, woraus sich vollkommenes künstlerisches (oder mystisches) Schaffen, vollkommenes Wissen und vollkommene Gesellschaftsordnung ergeben. Während der Westen dem folgerichtigen Rationalismus ganz verfallen ist und in der Philosophie nichts mehr zu sagen hat, kann das russische Volk eine Einheitsphilosophie aufbauen, die das Erbe von Ost und West übernehmen wird, weil es vom Rationalismus im Grund unverdorben ist.

Insoweit kann man den Ausführungen Solovjovs folgen, obschon sein 
System auf dem mystischen Erlebnis fusst, welches durchaus nicht einem jeden Menschen gegeben ist zu erleben. Später aber - schon in den Vorlesungen über das Gottmenschentum (I88o-8I) entwickelt Solovjov die Idee von der inkarnierten Weisheit Gottes und baut sein System ganz und gar auf seiner eigenen Vision der Sophia auf. Dies System sieht, von KobilinskiEllis in Tabellenform vorgeführt, wie folgt aus:

\begin{tabular}{|c|c|c|c|c|c|c|c|}
\hline I. Fides & $\begin{array}{l}\text { Sapien- } \\
\text { tia infusa }\end{array}$ & Theologie & $\begin{array}{l}\text { Die gött- } \\
\text { liche Tri- } \\
\text { nität }\end{array}$ & $\begin{array}{l}\text { Der Ur- } \\
\text { sprung } \\
\text { der Dinge }\end{array}$ & $\begin{array}{l}\text { Die gött- } \\
\text { liche } \\
\text { Offen- } \\
\text { barung }\end{array}$ & $\begin{array}{l}\text { Das Über- } \\
\text { seiende }\end{array}$ & Gott \\
\hline II. Sophia & $\begin{array}{l}\text { Sapientia } \\
\text { contemp- } \\
\text { lativa }\end{array}$ & $\begin{array}{l}\text { Sophio- } \\
\text { logie }\end{array}$ & $\begin{array}{l}\text { Die All- } \\
\text { einheit } \\
\text { des wahr- } \\
\text { haft } \\
\text { Seienden }\end{array}$ & $\begin{array}{l}\text { Das } \\
\text { Wesen } \\
\text { der Dinge }\end{array}$ & $\begin{array}{l}\text { Mystische } \\
\text { Kontem- } \\
\text { plation }\end{array}$ & $\begin{array}{l}\text { Die Uber- } \\
\text { natur }\end{array}$ & Himmel \\
\hline III. Ratio & $\begin{array}{l}\text { Sapientia } \\
\text { acquisita }\end{array}$ & $\begin{array}{l}\text { Philoso- } \\
\text { phie }\end{array}$ & $\begin{array}{l}\text { Die for- } \\
\text { melle Ein- } \\
\text { heit des } \\
\text { Seins }\end{array}$ & $\begin{array}{l}\text { Das ab- } \\
\text { strakte } \\
\text { Prinzip } \\
\text { der Dinge }\end{array}$ & $\begin{array}{l}\text { Rationelle } \\
\text { Spekula- } \\
\text { tion }\end{array}$ & $\begin{array}{l}\text { Die nat. } \\
\text { Vernunft }\end{array}$ & Mensch \\
\hline Ratio & & $\begin{array}{l}\text { Wissen- } \\
\text { schaft }\end{array}$ & $\begin{array}{l}\text { Die Viel- } \\
\text { heit der } \\
\text { Erschei- } \\
\text { nungen }\end{array}$ & $\begin{array}{l}\text { Die äusse- } \\
\text { re Form } \\
\text { der Dinge }\end{array}$ & $\begin{array}{l}\text { Sinnliche } \\
\text { Erfahrung }\end{array}$ & $\begin{array}{l}\text { Die } \\
\text { Natur }\end{array}$ & Weltseele $^{1}$ \\
\hline
\end{tabular}

„Das System Solowjevs“, sagt Madey, „erscheint uns als ein angelegter Versuch einer Synthese von christlichem Dogma und einem leuchtenden Wesen, das in der Welt Gestalt annimmt (inkarniert), um die Welt zu vergeistigen. Dieses Wesen hat den Namen Sophia tĥu the $\hat{u}^{\text {“' }}$. Wir sehen, das die wesenhafte Sophia einen zentralen Platz in dem System Solovjovs einnimmt. Sie unterscheidet sich von der Trinität dadurch, dass sie die Offenbarung der Trinität in der Region „Himmel“ vertritt. Sie unterscheidet sich von „Mensch" und von „Weltseele“ dadurch, dass sie eine höhere Stufe der Offenbarung vertritt. Sie ist aber als „Das wahrhaft Seiende“ nach Solovjov wirklich inkarniert, was dem Menschen die Möglichkeit bietet, mit ihr in persönliche Verbindung zu treten.

Die Vision der Sophia, der ewigen Weisheit Gottes, bedeutet für Vladimir Solovjov vor allem eine persönliche Offenbarung. Sie hat sich im Kulturleben Russlands in zwei verschiedenen Formen widergespiegelt, erstens in

\footnotetext{
${ }^{1}$ E. Kobilinski-Ellis, W. Solowjew, Monarchia Sancti Petri, Mainz 1929, S. 604 .

${ }^{2}$ Johannes Madey, Wladimir S. Solowjew und seine Lehre von der Weltseele, Paderborn I96I, S. III.
} 
der Philosophie, zweitens in der Poesie. In dem Bereich der Philosophie fusst Solovjors System auf einer religiösen Offenbarung, welche von der Kirche nicht gutgeheissen werden kann. Im Gegensatz zu dem Rationalismus der westlichen Philosophie, baut Solovjov sein idealistisches System auf, das aus dem „Einen“ hervorgeht (welches er ja die Fähigkeit hatte, aus persönlicher Erfahrung kennenzulernen), während der Rationalismus des Westens mit seinem Empirismus nur Einzelheiten der Untersuchung unterwerfen konnte und zu keinem einheitlichen Weltbild gelangte. Als Rückkehr zum Idealismus hatte Solovjovs System eine ungeheure Bedeutung für das russische Geistesleben der 8 oer Jahre. Schon in den 7oer Jahren war die Jugend des Materialismus und Rationalismus innerlich satt geworden. Sie kehrte dank des jetzt viel gelesenen Philosophen Peter Lavrovs ${ }^{1}$ allmählich zum Humanismus zurück, da ihr andere Werte als die materialistischen bewusst wurden. Die Bedeutung Lavrovs war aber vor allem eine praktische, indem Lavrov die Jugend zur Aufklärungstätigkeit unter den unteren Schichten des Volks anspornte. Beseelende Ideen, die den Zweck des Daseins erklärten, wurden aber erst von Solovjov in dieser Epoche unzweideutig ausgesprochen. Sie fanden denn auch grossen Anklang, jedenfalls als theoretische Lehre. Um aber verwirklicht zu werden, waren die Ideen Solovjovs zu sehr von seiner persönlichen Vision bewirkt. Sein System fusst auf der Inkarnation der göttlichen Weisheit, die es einem jeden nicht gegeben ist, kennenzulernen, es steht und fällt mit ihr. Eben deswegen konnte es keine Rückkehr zur Kirche zuwegebringen, obschon dies gerade sein Ziel war.

Solovjovs mystisches Erlebnis bleibt der orthodoxen Kirche wesensfremd. Denn die Inkarnation der Sophia ist eine Inkarnation des sog. ,"Taborlichts“, d. h. der Energie, oder einer der Energien, die von Gott ausstrahlen. In seinem Buch über Gregorios Palamas hebt der Metropolit Kiprian ausdrücklich hervor, dass I. „, die Energien nicht Kräfte der einen oder anderen Hypostase seien, sondern der ganzen Dreieinigkeit angehören“, dass 2. „die göttliche Energie, oder die Seite der Gottheit, die der Welt zugewandt ist, und für sich keine Hypostase ist" (also als selbständig nicht da ist)2. Damit

${ }^{1}$ Petr Lavrovič Lavrov (1823-1900), Philosoph positivistischer Richtung, veröffentlichte im Jahre I 870 sein unter der Jugend mit Enthusiasmus gelesenes Buch , Istoričeskija pis'ma“ unter dem Pseudonymen Mirtov zuerst erschienen in Nedelja I868-69, die den Historismus gegen Dm. Pisarev wieder zu Ehren kommen liessen.

${ }^{2}$ Kiprian, Archimandrit, Antropologija Sv. Grigorija Palamy, Pariž, I950, S. 290. 
wird von den Kirchenvätern auch die Lehre von der „Weltseele“, die in sich den Keim aller Seelen bergen sollte, abgelehnt. Die Sophia, die Weisheit Gottes ist keine Hypostase, kein von dem dreieinigen Gott verschiedenes Wesen, wie auch die Ideen der Dinge an und für sich keine selbständige Existenz besitzen, was übrigens auch von Gregorios Palamas ausdrücklich gesagt worden ist ${ }^{1}$.

Die Idee der inkarnierten Weisheit Gottes hat später u. a. Vater Sergej Bulgakov in seiner Trilogie „O Bogočelovečestve“ (Über das Gottmenschentum) stark beeinflusst, ist aber von Erzbischof Serafim Sobolev in ,Novoe učenie o Sofii Premudrosti Božiej“ neuerdings der Kritik der Kirche unterworfen worden. Dabei hat Serafim zum Ausgangspunkt seines Werkes die Worte des Apostels Paulus aus dem Korintherbrief I: 23-24 gewählt ,Wir aber predigen den gekreuzigten Christus, Gottes Kraft und Gottes Weisheit." Einen anderen Vermittler zwischen Gott und Mensch kennt die Kirche nicht. Erzbischof Serafim weist auch nach, dass alle Kirchen, die in Bulgarien oder in Russland anscheinend der heiligen Sophia geweiht waren, eben der zweiten Hypostase der Dreieinigkeit, d. h. Christus, und der nur von Ihm ausstrahlenden Weisheit geweiht waren, nicht also einer vierten „Gottheit“, die nicht existiert und nicht existieren kann².

Obschon die Kirche die Vision Solovjovs nicht gutheissen konnte, war sie machtlos, als es in den 8oer und goer Jahren galt, eine neue Religionsphilosophie in Russland zu begründen, deren Vertreter Florenskij, Merežkovskij und Berdjaev wurden, welche nach der Philosophie Solovjovs den festen Glauben an das eschatologisch sich nähernde „Dritte Reich des Geistes" verfochten. Sie fussten alle auf Solovjovs Lehre, dank welcher u. a. eine Rückkehr zu Kant in der Ethik, und eine Rückkehr zum deutschen Idealismus bewirkt wurde, als man nach Überwindung von Materialismus und Nihilismus zur Ergründung der zentralen Probleme des individuellen und gesellschaftlichen Menschen zurückkehrte. Die Vision der Alleinheit, der Sophia, steht im Hintergrund dieses neuen Denkens.

Ganz ausgesprochen wurde aber die Sophienlehre, als Lehre von der inkarnierten Weisheit Gottes, der hehren Jungfrau und Königin der Welt von der religiös gesinnten Gruppe der russischen Symbolisten aufgenommen.

1 Gregorius Palamas, MPGr i 50 col. I2 18.

2 Serafim Sobolev, Novoe učenie o Sofii Premudrosti Božiej, Sofija I935, S. І00-ıо . 
Obschon die Symbolisten (vielleicht nicht eidetisch begabt wie Solovjov) die Sophia selbst nie gesehen hatten, glaubten sie fest an ihr baldiges Kommen. Sie sahen in der Sophia die Vermittlerin zwischen Gott und Welt. Dies geht aus einem Briefe Alexander Bloks an Andrej Belyj u. a. sehr deutlich hervor. „Ich möchte sagen“, schreibt Blok, ,,dass ich Christus weniger liebe als „,Sie“ ".. Christus ist immer gut für „Sie“ aber ist dies nicht wesentlich. Das Licht der immerstrahlenden Neuen Göttin ist nämlich weder gut noch böse, sondern etwas mehr als dies ... Ich empfinde ,Sie“ als eine Stimmung, ja, meistens ist es so. Ich glaube, dass man „Sie“ sehen kann, aber nicht als eine leibliche Person, sondern vielmehr als Schatten in einem anderen Wesen und auch leblosen Dingen" "1. Mit diesem Glauben hängt die eschatologische, ,Solovjovsche“, Stimmung der Symbolisten zusammen. Die „Verteidigung des Guten“ und die übrige Philosophie Solovjovs ist den Symbolisten zu schwer. Sie würdigen aber in Solovjov den Poeten und den Propheten, den begnadigten Auserwählten der Königin der Welt, der Weltseele und des Reiches des Ewig-Weiblichen, des hehren „Dritten Reiches“ des Geistes.

${ }^{1}$ Aleksandr Blok i Andrej Belyj, ,Perepiska“, red. i vstup.stat'ja i kommentarii V. N. Orlova, Letopisi Gosudarstvennogo Literaturnogo Muzeja, 7, Moskva 1940, S. 35. - E. Benz, Die Vision. Erfahrungsformen und Bilderwelt, Stuttgart 1969, hat auch ein Kapitel über „Visionen der himmlischen Weisheit (der Jungfrau Sophia)“, wo Solovjovs Sophienbegegnungen behandelt sind [Korrekturnote, C.-M. Edsman]. 